\title{
Article \\ CaWRKY30 Positively Regulates Pepper Immunity by Targeting CaWRKY40 against Ralstonia solanacearum Inoculation through Modulating Defense-Related Genes
}

\author{
Ansar Hussain ${ }^{1}$, Muhammad Ifnan Khan ${ }^{1}$, Mohammed Albaqami ${ }^{2} \mathbb{D}$, Shahzadi Mahpara ${ }^{1}$, Ijaz Rasool Noorka ${ }^{1}$, \\ Mohamed A. A. Ahmed ${ }^{3}$, Bandar S. Aljuaid ${ }^{4}$, Ahmed M. El-Shehawi ${ }^{4}\left(\mathbb{D}\right.$, Zhiqin Liu ${ }^{5, *}$, Shahid Farooq ${ }^{6}$ \\ and Ali Tan Kee Zuan ${ }^{\text {,*(D) }}$
}

Citation: Hussain, A.; Khan, M.I.; Albaqami, M.; Mahpara, S.; Noorka, I.R.; Ahmed, M.A.A.; Aljuaid, B.S.; El-Shehawi, A.M.; Liu, Z.; Farooq, S.; et al. CaWRKY30 Positively Regulates Pepper Immunity by Targeting CaWRKY40 against Ralstonia solanacearum Inoculation through Modulating Defense-Related Genes. Int. J. Mol. Sci. 2021, 22, 12091. https://doi.org/10.3390/ijms222112091

Academic Editor: Maria R. Ercolano

Received: 29 September 2021

Accepted: 29 October 2021

Published: 8 November 2021

Publisher's Note: MDPI stays neutral with regard to jurisdictional claims in published maps and institutional affiliations.

Copyright: (c) 2021 by the authors. Licensee MDPI, Basel, Switzerland. This article is an open access article distributed under the terms and conditions of the Creative Commons Attribution (CC BY) license (https:// creativecommons.org/licenses/by/ $4.0 /)$.
1 Department of Plant Breeding and Genetics, Ghazi University, Dera Ghazi Khan 32200, Pakistan; ahtraggar@yahoo.com (A.H.); mifnan@yahoo.com (M.I.K.); smahpara@gudgk.edu.pk (S.M.); hod.pbg@gudgk.edu.pk (I.R.N.)

2 Department of Biology, Faculty of Applied Science, Umm Al-Qura University, Makkah 21955, Saudi Arabia; mmbaqami@uqu.edu.sa

3 Plant Production Department (Horticulture-Medicinal and Aromatic Plants), Faculty of Agriculture (Saba Basha), Alexandria University, Alexandria 21531, Egypt; mohamed_marie2009@yahoo.com

4 Department of Biotechnology, College of Science, Taif University, P.O. Box 11099, Taif 21944, Saudi Arabia; research@bssalj.com (B.S.A.); elshehawi@hotmail.com (A.M.E.-S.)

5 College of Crop Sciences, Fujian Agriculture and Forestry University, Fuzhou 350001, China

6 Department of Plant Protection, Faculty of Agriculture, Harran University, Şanlıurfa 63050, Turkey; csfa2006@gmail.com

7 Department of Land Management, Faculty of Agriculture, Universiti Putra Malaysia, Serdang 43400, Malaysia

* Correspondence: lzqfujian@126.com (Z.L.); tkz@upm.edu.my (A.T.K.Z.)

Abstract: The WRKY transcription factors (TFs) network is composed of WRKY TFs' subset, which performs a critical role in immunity regulation of plants. However, functions of WRKY TFs' network remain unclear, particularly in non-model plants such as pepper (Capsicum annuum L.). This study functionally characterized CaWRKY30 - a member of group III Pepper WRKY protein-for immunity of pepper against Ralstonia solanacearum infection. The CaWRKY30 was detected in nucleus, and its transcriptional expression levels were significantly upregulated by $R$. solanacearum inoculation (RSI), and foliar application ethylene (ET), abscisic acid (ABA), and salicylic acid (SA). Virus induced gene silencing (VIGS) of CaWRKY30 amplified pepper's vulnerability to RSI. Additionally, the silencing of CaWRKY30 by VIGS compromised HR-like cell death triggered by RSI and downregulated defense-associated marker genes, like CaPR1, CaNPR1, CaDEF1, CaABR1, CaHIR1, and CaWRKY40. Conversely, transient over-expression of CaWRKY30 in pepper leaves instigated HR-like cell death and upregulated defense-related maker genes. Furthermore, transient over-expression of CaWRKY30 upregulated transcriptional levels of CaWRKY6, CaWRKY22, CaWRKY27, and CaWRKY40. On the other hand, transient over-expression of CaWRKY6, CaWRKY22, CaWRKY27, and CaWRKY40 upregulated transcriptional expression levels of CaWRKY30. The results recommend that newly characterized CaWRKY30 positively regulates pepper's immunity against Ralstonia attack, which is governed by synergistically mediated signaling by phytohormones like ET, ABA, and SA, and transcriptionally assimilating into WRKY TFs networks, consisting of CaWRKY6, CaWRKY22, CaWRKY27, and CaWRKY40. Collectively, our data will facilitate to explicate the underlying mechanism of crosstalk between pepper's immunity and response to RSI.

Keywords: chili pepper (Capsicum annuum); Ralstonia solanacearum; CaWRKY30; WRKY TFs

\section{Introduction}

Plants frequently get exposed to various biotic and abiotic stresses during their life span due to their immobile nature [1,2]. Plants have developed several defense mechanisms in response to repeated selection pressure from major ecological and environmental 
constraints. Different transcriptional factors (TFs) interconnect to develop sophisticated transcriptional networks for regulating these classic defense mechanisms at a transcriptional level $[3,4]$. Defense reactions to various stresses are properly synchronized and controlled as they are critical for plants in the form of energy expenditure and development [5]. The defense mechanisms might vary in different plant species because of varied environmental circumstances affecting their acclimation [6-8]. Hence, defense system employed by model plants cannot be fully implied to non-model plants. Therefore, defense systems of plants have been a prime focus during past decades; however, these studies were mostly focused on model plants, i.e., Arabidopsis thaliana and rice (Oryza sativa L.). Nevertheless, functional synchronization of these transcriptional complexes to regulate plants' response to various stresses has been poorly investigated, mainly in non-model plants.

The WRKY proteins comprise of the largest TFs' family in plants. The WRKY TFs contain one or two WRKY domains and commonly WRKYGQK sequence at the N-terminus followed by $\mathrm{C} 2 \mathrm{HC}$ or $\mathrm{C} 2 \mathrm{H} 2$ zinc finger motif $[4,9,10]$. The WRKY proteins are phylogenetically divided into three main groups (i.e., group I-III) based on number of WRKY domains and structure of zinc-finger motif. The group II of WRKY TFs is again subdivided into five sub-groups (IIa, Ilb, IIc, IId, and IIe) [11-13]. The WRKY members mainly bind with $\mathrm{W}$-boxes [TTGAC $(\mathrm{C} / \mathrm{T})]$ found in promoter regions of various target genes by inserting an exclusive wedge vertically into DNA's major groove [14] by WRKY GQK motif on the second $\mathrm{b}$ strand, which stimulates transcriptional modulation of the expression of target genes [14-16]. The WRKYs have been involved in several biological systems in plants, including seed dormancy, seed germination, senescence, and seed development. Similarly, these are also involved in response to biotic and abiotic stresses and switching transcription of their target genes [17-20].

It is reported that a group of WRKY TFs or one WRKY TF transcriptionally induced by only one stress modulates several stresses. The W-boxes are enriched inside promoter regions [21-24]. These studies depict the presence of WRKY networks implicated in response to a particular stress, or a combination of two or more. The role of WRKY proteins and underlying mechanisms in response to biotic and abiotic stresses have been frequently investigated in the past decade; however, emphasis of these studies was primarily on one gene in response to one stress in model plants, e.g., A. thaliana and $O$. sativa. Recently, an important functional variance among close homologs of WRKY TFs from various plant species has been suggested [24,25]. The functions of WRKYs and WRKY web in non-model plants such as Capsicum annuum L., in response to single or combined stresses, has been poorly investigated until now.

Pepper is globally known for its economic importance. It is sown in uplands during temperate seasons [26,27]. Pepper is exposed to various soil-borne diseases such as pepper blight and bacterial wilt caused by bacterial pathogens, i.e., Phytophthora capsica and Ralstonia solanacearum, respectively [28,29]. The coincidence of these pathogens imparts devastating impacts on pepper production under high temperature and high humidity (HTHH). The HTHH impairs pepper's immunity mediated by R protein and speeds up pathogen growth and development. Furthermore, pathogen attack and HTHH simultaneously exert natural selection pressure on pepper, which has affected pepper's evolution in the past [30-32]. Conversely, simultaneous occurrence of pathogen infection and HTHH might not affect evolution of model plants, like $A$. thaliana and $O$. sativa. Hence, pepper seems more appropriate for research regarding immunity against biotic or abiotic stresses, i.e., RSI or HTHH.

Ralstonia solanacearum causes bacterial wilt in numerous crops, i.e., pepper, tomato, potato, etc. It is a soilborne gram-negative $\beta$-proteobacterium [33]. It forms rapid colonies in the xylem tissues, which leads to bacterial wilt disease in the infested plants [34].

The responses of plant species to biotic and abiotic stress are known to be regulated by phytohormones [35]. Phytohormones are tiny molecules involved in plant growth regulation, plant reproduction, and survival. Salicylic acid (SA) and jasmonic acid (JA) 
are critical for immune responses of plant species. These two are regarded as critical foundations of the immune responses of plants against pathogens. Biosynthesis and signaling of SA is vital for defense against biotrophic pathogens, while JA provides defense against necrotrophic pathogens $[36,37]$. Ethylene (ET) assists in defense responses against several plant-pathogen interactions [37].

Various indigenous pepper cultivars of subtropical areas demonstrate accelerated disease resistance, even under HTHH [38]. Heat related cis-element HSE generally coexist with phytohormones, including SA, JA, ET, ABA, or pathogen related cis-elements in promoter regions of most MAPKs and CDPKs involved in plant immunity [39-41]. It recommends presence of crosstalk between immunity and HTHH. Pepper genome is 27 and 7.5 times bigger as compared to $A$. thaliana and $O$. sativa, respectively. However, it has only 73 WRKY genes, whereas smaller genomes of $A$. thaliana and O. sativa have 72 and 122 WRKY genes, respectively [9,42,43]. We previously detected that CaWRKY6 [44], CaWRKY22 [9], CaWRKY40 [45], and CaWRKY58 [46] have been involved in pepper's

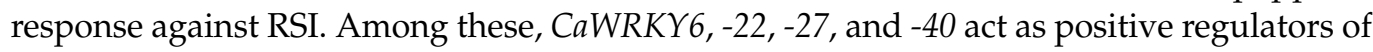
plant immunity, whereas CaWRKY58 is a negative regulator. These genes possess subset of HSE elements and W-boxes in their promoter region and in the promoters of other WRKY TFs, inferring the role of WRKY networks in regulation of pepper's response against RSI. Additionally, CaWRKY40 was directly regulated by CaWRKY6 [44] and CabZIP63 [16], while CaWRKY40 was indirectly regulated by CaCDPK15 [47]. Conversely, most of the pepper's WRKY TFs have not been characterized yet for its response to pathogen attack.

In the current study, a full-length cDNA for WRKY TFs family of pepper was isolated and named CaWRKY30. We characterized it expressionally and functionally and found that CaWRKY30was induced by RSI and foliar application of SA, JA, ET, and ABA. Transient over-expression of CaWRKY30 in pepper leaves triggered HR-like cell death and $\mathrm{H}_{2} \mathrm{O}_{2}$ production. Silencing of CaWRKY30 in pepper compromised the immunity of pepper to RSI. These results suggest that CaWRKY30 positively regulates pepper's immunity to RSI.

\section{Results}

2.1. Cloning and Sequence Analysis of CaWRKY30

A new WRKY gene CaWRKY30 (CA01g34480) was identified by genome wide analysis (http:/ / passport.pepper.snu.ac.kr (accessed on 25 October 2021). The CaWRKY30 gene was chosen for functional characterization, since the existence of immunity-related cis elements such as TGA (TGACG motif-binding factor), TGACG-motif, TATC-box, TATAbox, and W-box in the promoter region designate its possible role in pepper's immunity (Figure S1). A cDNA fragment of CaWRKY30 (CA01g34480) of 924 bp open-reading-frame (ORF) was cloned by using gene specific primers (Table S1). The length of deduced amino acid sequence of CaWRKY30 was 307 amino acids residues, possessing conserved WRKY domain, and it was categorized into group III (Figure 1). The predicted protein has a size and theoretical pI of $34.55 \mathrm{Kda}$ and 7.32, respectively. The CaWRKY30 shares 88, 60, 68, and 57\% amino acid identity with NsWRKY 53, CcWRKY53, NaWRKY53, and StWRKY53, respectively (Figure S2).

\subsection{Transcriptional Expression of CaWRKY30 under RSI and Treatment with Various Phytohormones}

The presence of immunity-associated cis-elements in the promoter region of CaWRKY30 indicated its potential role in pepper's immunity RSI. To check the notion, qRT-PCR analysis was carried out to assess the transcriptional levels of CaWRY30 upon RSI. The transcriptional levels of CaWRKY30 were increased in Ralstonia-treated leaves compared to mock-treated leaves (Figure 2A). The enhanced CaWRKY30 transcriptional expression levels were consistent between $0 \mathrm{~h}$ to $48 \mathrm{~h}$ post treatment (hpt). The highest transcriptional expression levels were recorded at $48 \mathrm{hpt}$ (Figure 2A). 

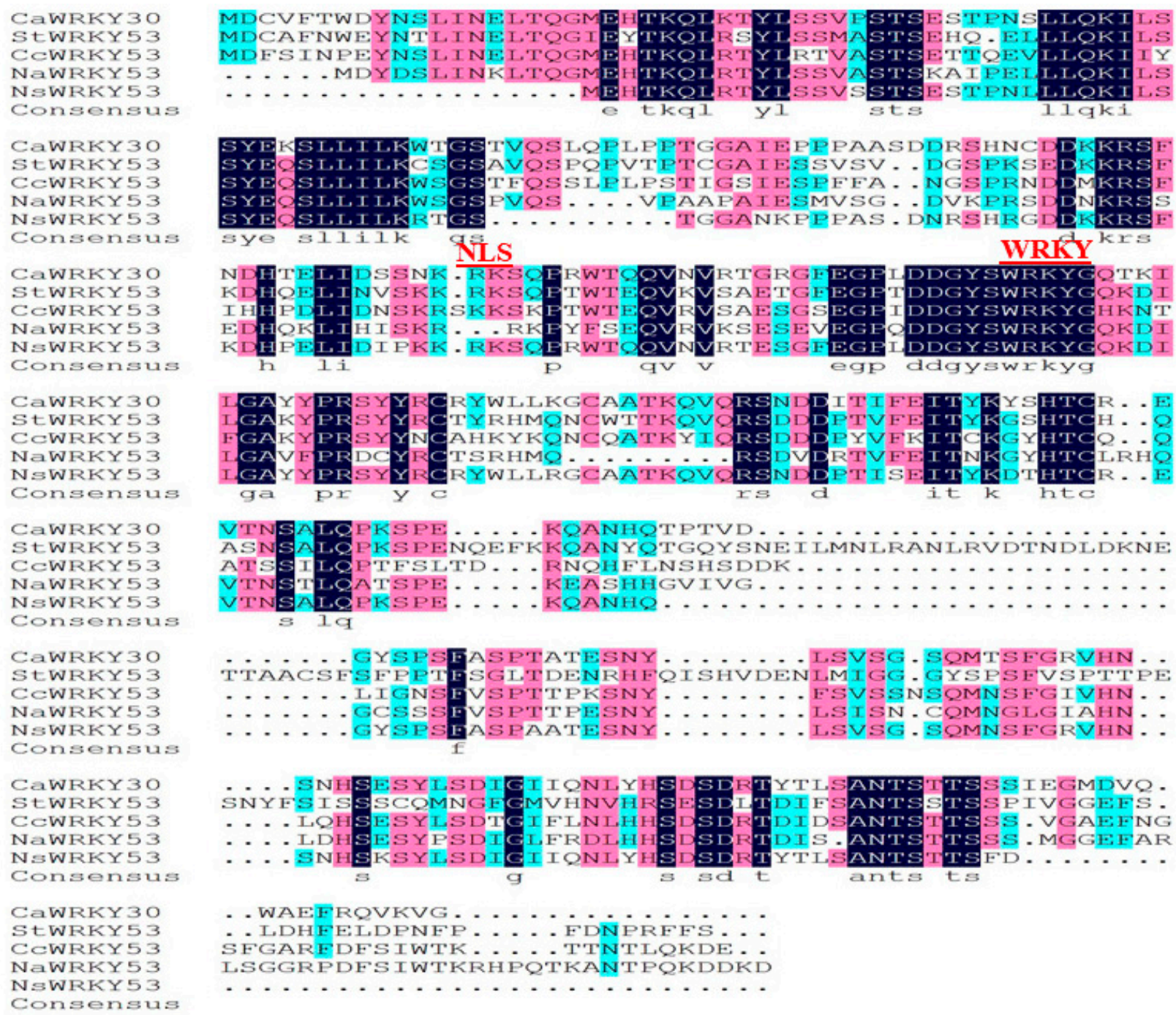

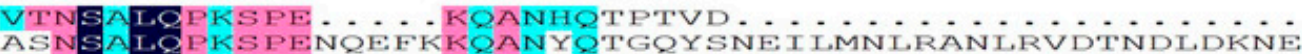

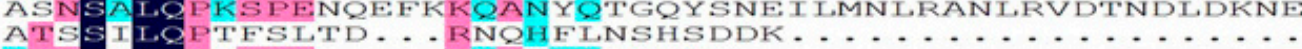
VTNSTIQATSPE

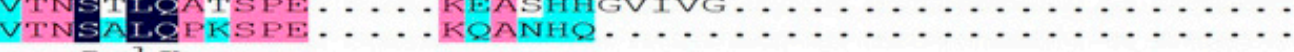

- . GYSPSEASPTATESNY - . . . LSVSG - SQMTSEGRVHN TTAACSESFPRTESGITDENRHFQISHVDENIMIGG - GYSPSEVSPTTPE $\ldots . . .$. IIGNSEVSPTTPKSNY ...... ESVSSNSQMMNSFGIVHN.

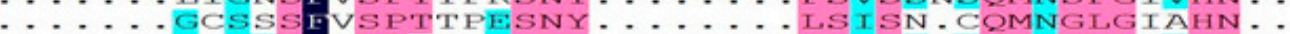

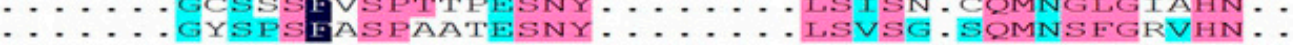

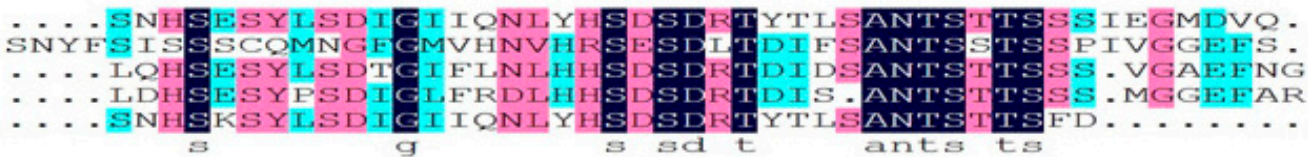

. WAEFRQVKVG.........................

- LDHFELDPNFP.... . FDNPRETS..

LSGGRPDFSIWTKRHPQTKANTPQKDDKD

Figure 1. Structural analysis of CaWRKY30. Comparison of CaWRKY30 deduced amino acid sequence with representative related proteins from Solanum tuberosum StWRKY53 (XP_015166339.1), Capsicum chinese CcWRKY53 (PHU08488.1), Nicotiana attenuate NaWRKY53 (XP_019233122.1), and Nicotiana sylvestris NsWRKY53 (XP_009768977.1). Green shading indicates 50-75\% identity, pink shading depicts 75-100\% identity, and blue shading indicates 100\% identity. Analysis was assayed by using DNAMAN5.

Phytohormones, including ET, ABA, SA, and JA mediated signaling pathways, are key regulators of plant reactions to biotic and abiotic stresses. The regulation of CaWRKY30 by phytohormones-mediated signaling pathways was studied by foliar application of ET, ABA, SA, and JA using qRT-PCR analysis. The results indicated that relative transcriptional expression levels of CaWRKY30 were enhanced from $0 \mathrm{~h}$ to $48 \mathrm{hpt}$ with $100 \mu \mathrm{M}$ ET as compared to mock. These transcriptional levels were highest at $48 \mathrm{hpt}$ with ET (Figure 2B). Foliar application of $100 \mu \mathrm{M}$ ABA significantly increased transcriptional levels of CaWRKY30 as compared to mock. Transcriptional levels reached to the highest level at $12 \mathrm{hpt}$ with ABA (Figure 2C). Results indicated that transcriptional accumulation of CaWRKY30 was significantly increased after application of $1 \mathrm{mM} \mathrm{SA}$, as compared to mock. These enhanced transcriptional expression levels were highest at $24 \mathrm{hpt}$ (Figure 2D). 
A

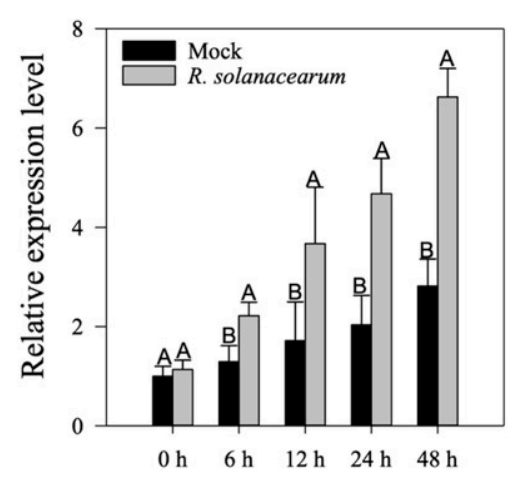

$\mathrm{C}$

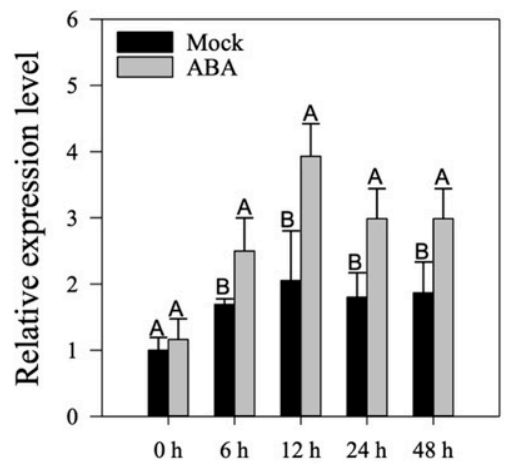

B

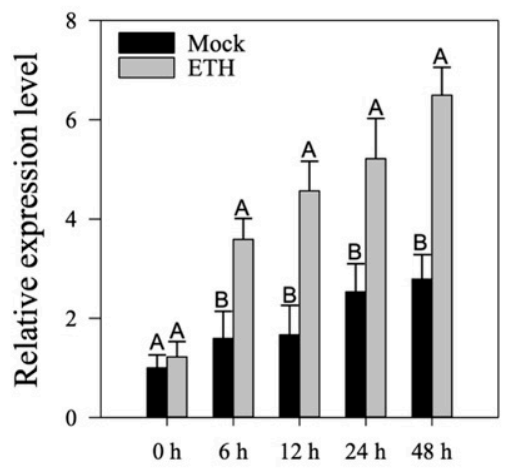

$\mathrm{D}$

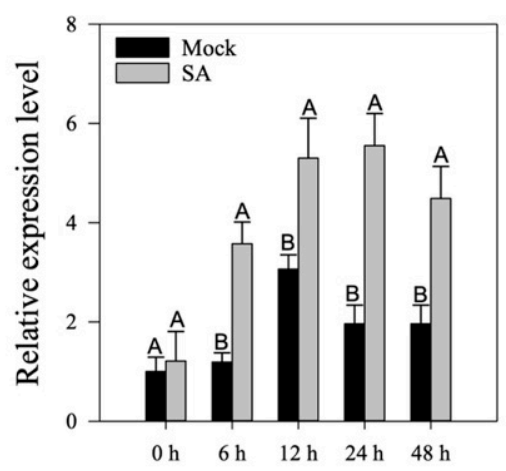

Figure 2. The qRT-PCR assay of CaWRKY30 relative transcriptional expression levels in pepper leaves subjected to Ralstonia solanacearum inoculation (RSI), and foliar application of various phytohormones. The qRT-PCR analysis was used to check the transcriptional levels of CaWRKY30 in pepper leaves subjected to various treatments including RSI (A), application of $100 \mu \mathrm{m}$ ET (B), application of $100 \mu \mathrm{m} \mathrm{ABA} \mathrm{(C),} \mathrm{and} \mathrm{application} \mathrm{of} 1 \mathrm{mM} \mathrm{SA}$ (D) at different time intervals. The transcriptional abundance in RSI-treated leaves was compared with $\mathrm{MgCl}_{2}$-treated control leaves (mock), whose relative expression level was set to "1". (B-D) The transcriptional expression levels in phyto-hormone-treated leaves were compared with $\mathrm{ddH}_{2} \mathrm{O}$-treated leaves (mock), whose expression level was set to " 1 ". The height of the bar indicates means, and error bars indicate the standard error of means. Different letters above the bars show a significant difference between the means based on the Fisher's protected LSD test.

\subsection{CaWRKY30 Was Found to Be Localized in Nucleus}

Sequence analysis by using WoLFPSORT ("http://www.genscript.com/psort/wolf_ psort.html \T1 \textquotedblrightolf_psort.htm (accessed on 29 October 2021)) depicted that predicted CaWRKY30 protein sequence possesses a putative nuclear localization signal (Figure 1), showing its potential targeting in nucleus. To validate this perception, a CaWRKY30 GFP fusion construct was generated that was driven by constitutive promoter of CaMV35S. Afterwards, this constituted vector was transferred into Agrobacterium strain GV3101. We transiently over-expressed CaWRKY30 GFP construct in leaves of Nicotiana benthamiana by A. tumefaciens injection, and GFP signals were examined by confocal fluorescence microscope. Results depicted that GFP signals of CaWRKY30-GFP was present in the nuclei, while GFP of control was detected in various subcellular portions, like cytoplasm and nuclei (Figure 3). 


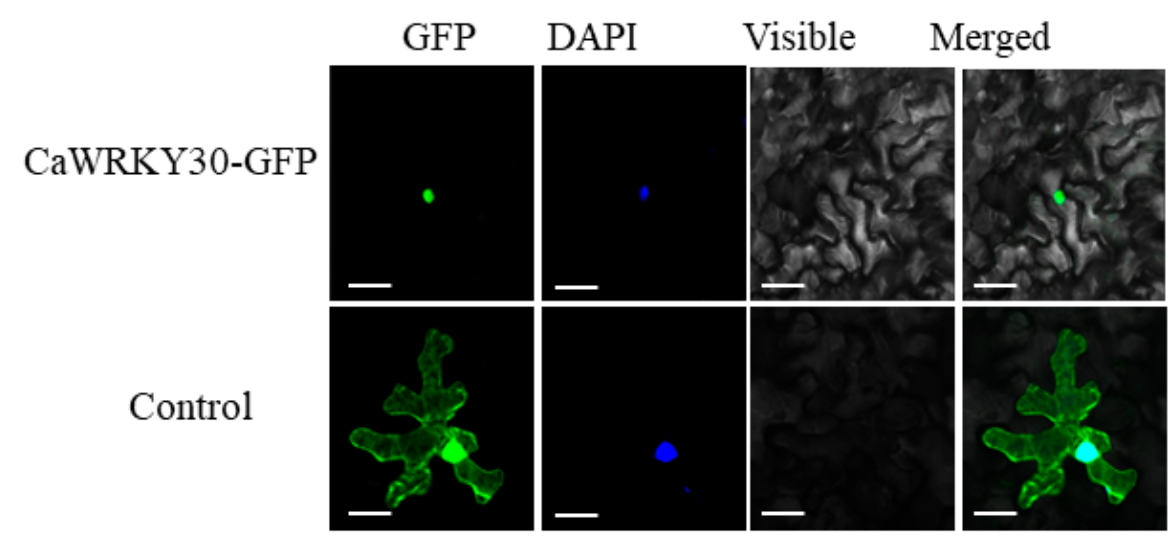

Figure 3. Subcellular localization of CaWRKY30. The CaWRKY30 was fully located in the nucleus of Nicotiana benthamiana leaves. Green color demonstrates GFP. Blue color demonstrates DAPI staining of nucleus. Cyan color demonstrates fusion of green GFP and blue DAPI stained nucleus. GFP signal (Green) for the control N. benthamiana leaves was detected all over the cell. Images were captured by confocal microscopy at $48 \mathrm{~h}$ post inoculation. Bars $=25 \mu \mathrm{m}$.

\subsection{Silencing of CaWRKY30by VIGS Curtailed Pepper's Immunity to RSI and Downregulated Immunity Associated Marker Genes}

The CaWRKY30 was silenced by virus induced gene silencing (VIGS) to study its role in pepper's immunity. A total of 50 CaWRKY30-unsilenced (TRV:00) and 50 CaWRKY30silenced (TRV:CaWRKY30) plants were acquired. Six CaWRKY30 silenced plants were randomly selected to check their efficiency of gene silencing by root inoculation with cells of compatible virulent $R$. solanacearum strain. Our findings indicated that transcriptional levels of CaWRKY30 were decreased by 30\% in Ralstonia-treated CaWRKY30 silenced plants as compared to unsilenced plants, validating the silencing of CaWRKY30 (Figure 4A). The CaWRKY30 silenced plants showed significantly higher susceptibility to RSI as compared to unsilenced plants. Pepper's susceptibility to pathogen was increased by showing a rise in Ralstonia population growth, marked by higher cfu values in CaWRKY30 silenced pepper plants as compared with unsilenced plants at $3 \mathrm{~d}$ and 5 days post inoculation (dpi) (Figure 4B). Histochemical staining assay was done to detect the $\mathrm{H}_{2} \mathrm{O}_{2}$ production and cell necrosis in Ralstonia inoculated CaWRKY30 silenced (TRV:CaWRKY30), and unsilenced (TRV:00) pepper leaves. A dark DAB staining (sign of $\mathrm{H}_{2} \mathrm{O}_{2}$ production) and $\mathrm{HR}$-like cell death indicated by dark trypan blue staining was noticed in unsilenced pepper leaves at 48 hpi (hours post inoculation). Conversely, the concentrations of trypan blue and DAB staining were significantly decreased in CaWRKY30 silenced pepper leaves (Figure 4C). Electrical conductivity - an indicator of ion leakage - was calculated to study plasma membrane damage and cell death after RSI. The results exhibited that unsilenced plants treated with RSI exhibited higher ion leakage than Ralstonia-treated CaWRKY30 silenced plants at 24 and 48 hpi (Figure 4D). Relative disease index was estimated up to $10 \mathrm{dpi}$ for inferring the level of disease in CaWRKY30-silenced and un-silenced plants after RSI (Supplementary Table S2). Prominent disease symptoms were noticed in CaWRKY30 silenced plants at $10 \mathrm{dpi}$, while unsilenced plants expressed little disease symptoms (Figure 4E). Six CaWRKY30-silenced and un-silenced plants were randomly chosen and infiltrated with $R$. solanacearum in the roots for phenotype assay. Obvious wilting disease symptoms were noticed in CaWRKY30 silenced pepper at $10 \mathrm{dpi}$, while unsilenced plants expressed very feeble wilting symptoms (Figure 4F). The qRT-PCR analysis was carried out to study the transcriptional accumulation of immunity related marker genes and results expressed that transcriptional expression levels of immunity associated marker genes such as CaPR1, CaNPR1, CaDEF1, CaABR1, and CaHIR1 were reduced in CaWRKY30-silenced plants as compared to un-silenced plants at 48 hpi (Figure $4 \mathrm{G}$ ). 


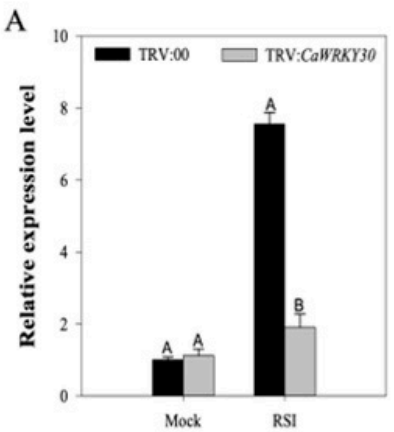

E

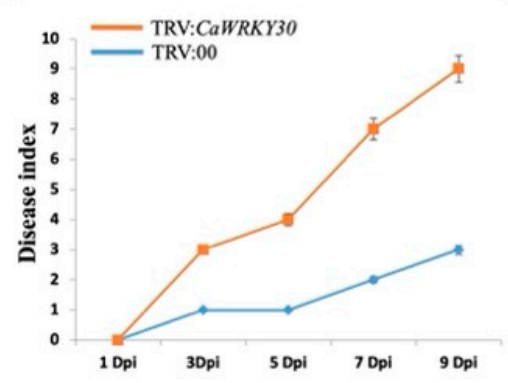

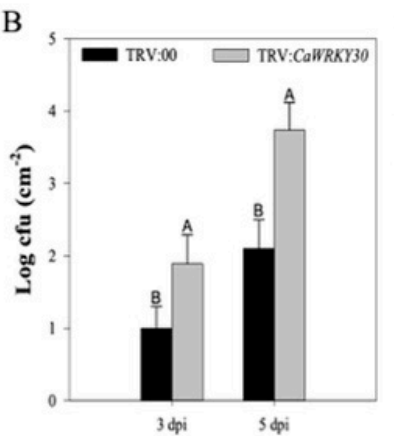

F
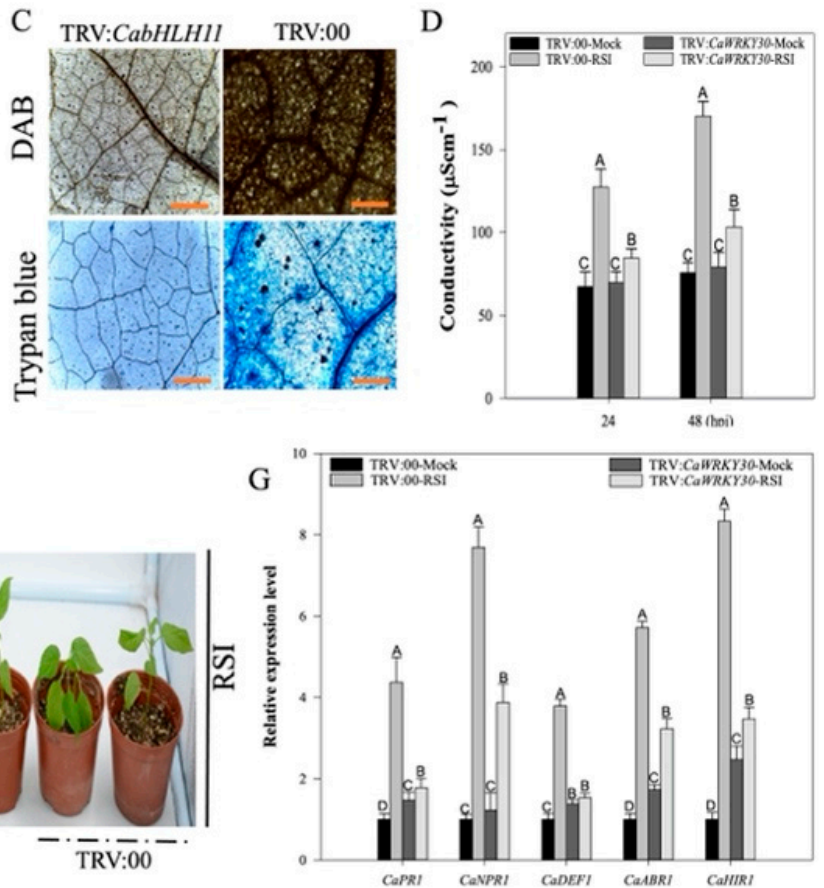

Figure 4. Silencing of CaWRKY30 by VIGS curtailed pepper's resistance to RSI and upregulated immunity associated marker genes. The qRT-PCR assay of CaWRKY30 expressional levels in R. solanacearum-inoculated, mock (inoculated with $\mathrm{MgCl}_{2}$ solution) CaWRKY30-silenced pepper plants (TRV:CaWRKY30), and control plants (TRV:00) (A). Difference in growth of $R$. solanacearum between CaWRKY30-silenced and un-silenced (control) pepper plants inoculated with $R$. solanacearum at 3 and 5 dpi (B). Histochemical staining (DAB and trypan blue staining) in R. solanacearum-inoculated CaWRKY30-silenced (TRV:CaWRKY30) and un-silenced (TRV:00) pepper leaves at $48 \mathrm{hpi}$. Scale bar $=50 \mu \mathrm{m}$ (C). Electrolyte leakage measurement as ion conductivity to evaluate the cell-death responses in the leaf discs of CaWRKY30-silenced (TRV:CaWRKY22) and un-silenced (TRV:00) pepper plants at 24 and 48 hpi with and without $R$. solanacearum (D). CaWRKY30-silencing by VIGS enhanced pepper plants susceptibility to $R$. solanacearum infection. Disease index significantly escalated with the passage of time. CaWRKY30-silenced (TRV:CaWRKY30) plants expressed high susceptibility to R. solanacearum infection as compared to un-silenced (TRV:00) plants with the passage of time. Averages are based on four biological replicates with five plants per replication (E). Phenotypic effect of $R$. solanacearum treatment on CaWRKY30-silenced (TRV:CaWRKY30) and un-silenced (TRV:00) pepper plants at $10 \mathrm{dpi}$ (F). qRT-PCR assay of transcriptional levels of defense-associated marker genes in CaWRKY30-silenced (TRV:CaWRKY30) and un-silenced (TRV:00) pepper plants at $48 \mathrm{~h}$ post inoculation with $R$. solanacearum (G). The relative transcriptional expression level of mock-treated un-silenced plants was set to " 1 ". The height of the bar indicates means and error bars indicate the standard error of means. Data represents the means $\pm \mathrm{SE}$ from four biological replicates. Different letters above the bars show significant differences among means.

2.5. CaWRKY30 Transient Over-Expression Stimulated HR-Like Cell Death, Production of $\mathrm{H}_{2} \mathrm{O}_{2}$, and Transcriptional Upregulation of Immunity Related Marker Genes

The VIGS experiments indicated that CaWRKY30 positively regulates pepper's immunity to RSI. Transient over-expression experiments were performed by infiltrating GV3101 cells carrying 35S:00 (EV) or 35S:CaWRKY30 into pepper leaves for further validation of this speculation. We studied the role of CaWRKY30 transient over-expression on induction of HR-like cell death and $\mathrm{H}_{2} \mathrm{O}_{2}$ accumulation in pepper plants. The expression of CaWRKY30 in pepper plants was established by western blotting experiments (Figure 5A). The HR like cell death and $\mathrm{H}_{2} \mathrm{O}_{2}$ accumulation was confirmed by trypan blue and DAB staining, respectively. Results revealed that transient over-expression of CaWRKY30 caused HR-like cell death and $\mathrm{H}_{2} \mathrm{O}_{2}$ accumulation in pepper leaves manifested by darker trypan blue staining and dark brown DAB staining (Figure 5B). Conductivity was performed to study the intensity of cell necrosis caused by transient over-expression of CaWRKY30, and results indicated that pepper leaves transiently over-expressing CaWRKY30 showed more ion leakage at 24 and $48 \mathrm{~h}$ after Agro-infiltration as compared with pepper leaves over-expressing 
empty vector (Figure 5C). Relative transcriptional expression levels of defense-associated marker genes such as ET-biosynthesis related CaPR1, SA-related CaNPR1, ABA-responsive CaABR1, and HR-associated marker gene CaHIR1 were detected by qRT-PCR analysis.

Our results depicted that transient over-expression of CaWRKY30 in pepper leaves notably amplified transcriptional accumulation of CaPR1, CaNPR1, CaDEF1, CaABR1, and CaHIR1 in pepper as compared to transiently over-expressing empty vector pepper plants at $48 \mathrm{hpi}$ (Figure 5D). These results portray that CaWRKY30 acts as a positive regulator of plant $\mathrm{HR}$-like cell death, $\mathrm{H}_{2} \mathrm{O}_{2}$ production, and transcriptional upregulation of immunity related marker genes.

A
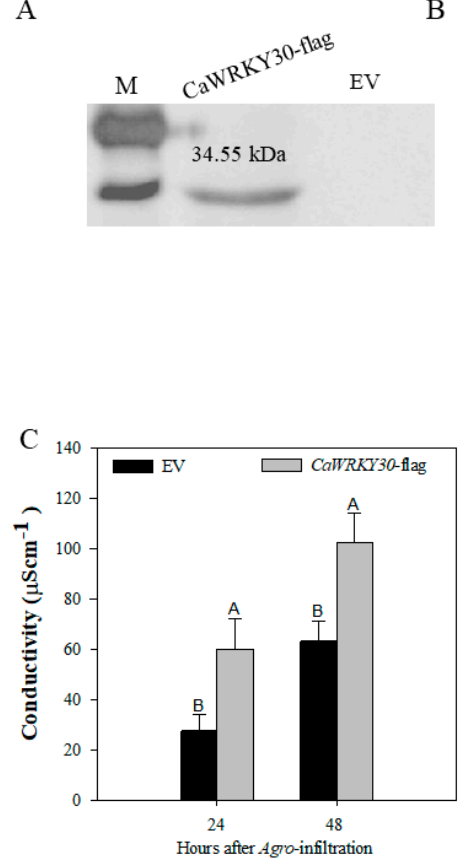

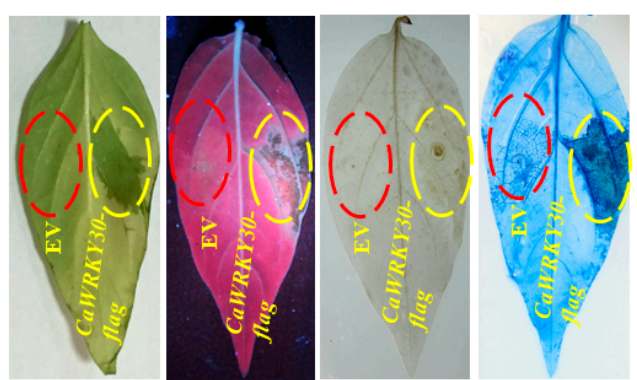

$\mathrm{D}$

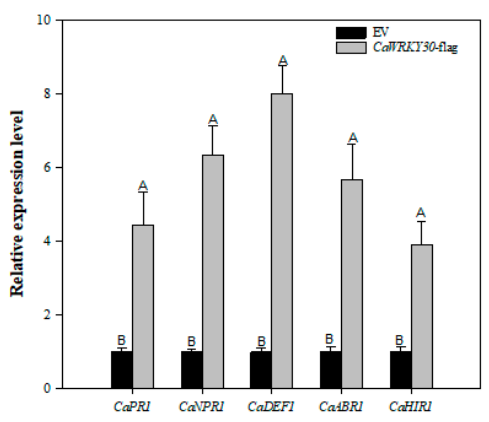

Figure 5. Transient over-expression of CaWRKY30 induced HR-like cell death, $\mathrm{H}_{2} \mathrm{O}_{2}$ accumulation, and expression of immunity-associated marker genes in pepper leaves. Successful over-expression of CaWRKY30-Flag was confirmed by western blotting experiment (A), HR-like cell-death triggered by transient over-expression of 35S:CaWRKY30, confirmed by phenotype detection, exposure under UV light, and DAB and Trypan Blue staining at $48 \mathrm{hpi}$, respectively; Scale bar $=50 \mu \mathrm{m}$ (B), Measurement of electrolyte leakage (ion conductivity) to estimate the cell death response in pepper leaf discs at 24 and $48 \mathrm{~h}$ after agro-infiltration, respectively (C), qRT-PCR assay to check the transcriptional expression levels of immunity-associated marker genes, including CaPR1, CaNPR1, CaDEF1, CaABR1, and CaHIR1 in 35S: CaWRKY30 expressed pepper leaves at $48 \mathrm{hpi}$, respectively (D). The relative expression level of known defense-related marker-genes in pepper leaves transiently over-expressing the empty vector was set to "1". Data representing the means \pm SE from four biological replicates. Error bars are standard errors of the means. Different letters above the bars indicates significant differences among the means.

\subsection{Inter-Relationship between CaWRKY30 and CaWRKY40}

It is reported that different WRKY TFs form networks together and we previously reported that various members of WRKY TFs, i.e., CaWRKY6, CaWRKY22, and CaWRKY40, are expressionally and functionally linked to each other. Therefore, we postulate that CaWRKY30 might relate to other members of the WRKY family, including CaWRKY40, which is a positive regulator of pepper's immunity against RSI. The qRT-PCR analysis was performed for further investigation of possible feedback regulation of CaWRKY40 by CaWRKY30, and to study the potential modulation of CaWRKY30 by CaWRKY40 transient over-expression assay or by silencing of CaWRKY 40 by VIGS. 
Our results expressed transcriptional levels of CaWRKY30 in pepper leaves transiently over-expressing CaWRKY40 increased at 24 and 48 hpi in comparison with plants transiently over-expressing empty vector (EV) (Figure 6A), whereas the transcriptional levels of CaWRKY40 increased in those plants transiently over-expressing CaWRKY 30 as compared to plants transiently over-expressing EV (Figure 6B). Contrastingly, our results depicted that transcriptional accumulation of CaWRKY40 was notably downregulated in Ralstonia-infected CaWRKY30-silenced plants as compared to un-silenced plants (Figure 6C). Additionally, transcriptional accumulation of known immunity linked marker genes, e.g., CaPR1, CaNPR1, CaDEF1, CaABR1, and CaHIR1 was fully or partially suppressed in CaWRKY30 silenced plants that was triggered by CaWRK40 (Figure 6D).

This data indicates transcriptional regulation of CaWRKY40 by CaWRKY30 and the presence of positive regulatory loop involving CaWRKY30 and CaWRKY40.
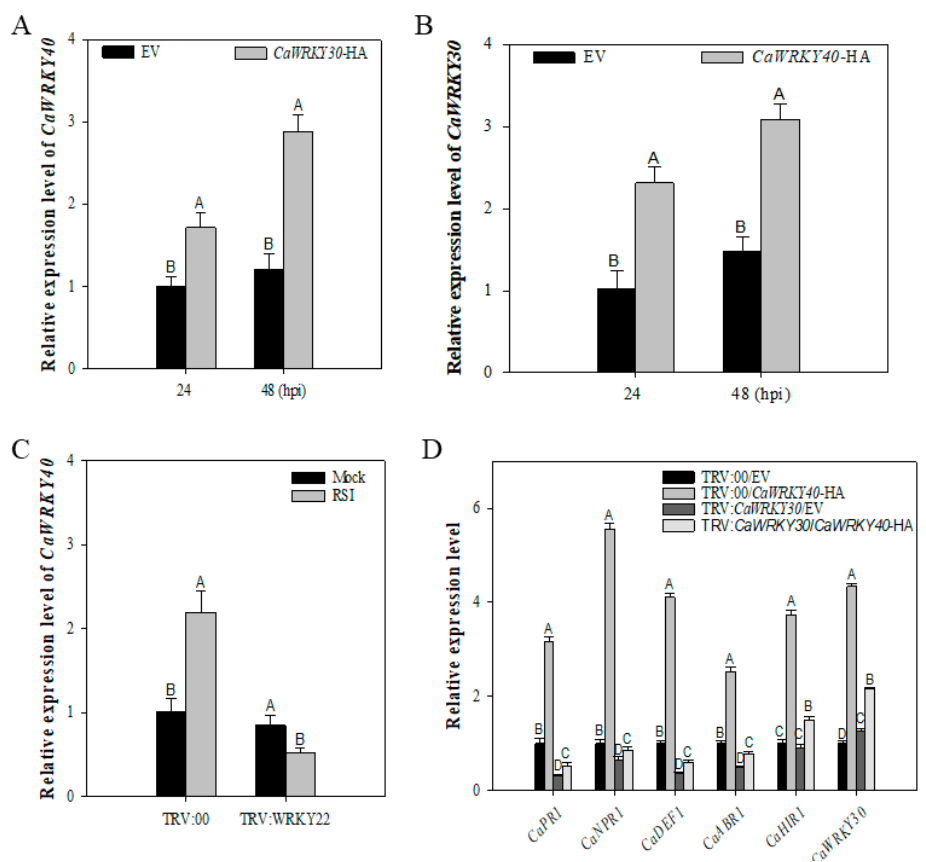

Figure 6. Inter-relationship between CaWRKY30 and CaWRKY40. Transcriptional expression levels of CaWRKY40 in pepper's leaves transiently over-expressing CaWRKY30 at a time interval of 24 and 48 hpi (A), Transcriptional expression levels of CaWRKY30 in pepper's leaves transiently over-expressing CaWRKY40 at time interval of 24 and 48 hpi (B), qRT-PCR analysis of CaWRKY40 transcriptional expression levels inCaWRKY30-silenced and un-silenced pepper plants at 48 hpi (C), qRT-PCR assay of the transcriptional levels of defense-associated marker genes in CaWRKY30silenced and un-silenced pepper plants transiently over-expressing 35S: CaWRKY40-HA and 35S:00 at $48 \mathrm{hpi}$ (D). The data are means $\pm \mathrm{SE}$ from four biological replicates. Error bars are standard errors of the means. Different letters above the bars show significant differences among means.

\subsection{Inter-Relationship among CaWRY30 and CaWRKY6, CaWRKY22, and CaWRKY27}

In our previous studies, CaWRKY 40 was detected to be associated expressionally and functionally to different WRKY TFs, such as CaWRKY6 and CaWRKY22. The relationship among CaWRKY30 and CaWRKY40 indicates that CaWRKY30 is possibly linked with different WRKY TFs involved in pepper's immunity against RSI. To confirm this assumption, qRT-PCR analysis was performed to study relationship among CaWRKY30 and different WRKY TFs which have been previously reported to be involved in pepper's immunity against RSI, including CaWRKY6, CaWRKY22, and CaWRKY27. Our results indicated that transcriptional accumulation of CaWRKY30 was amplified in CaWRKY6, CaWRKY22, and CaWRKY27 transiently over-expressing pepper plants at 48 hpi (Figure 7A), while transcriptional accumulation of CaWRKY6, CaWRKY22, and CaWRKY27 were also increased in pepper leaves transiently over-expressing CaWRKY30 at 48 hpi (Figure 7B). Our results 
of qRT-PCR assay imply that CaWRKY30 and CaWRKY6, CaWRKY22, and CaWRKY27 are expressionally and functionally inter-related.
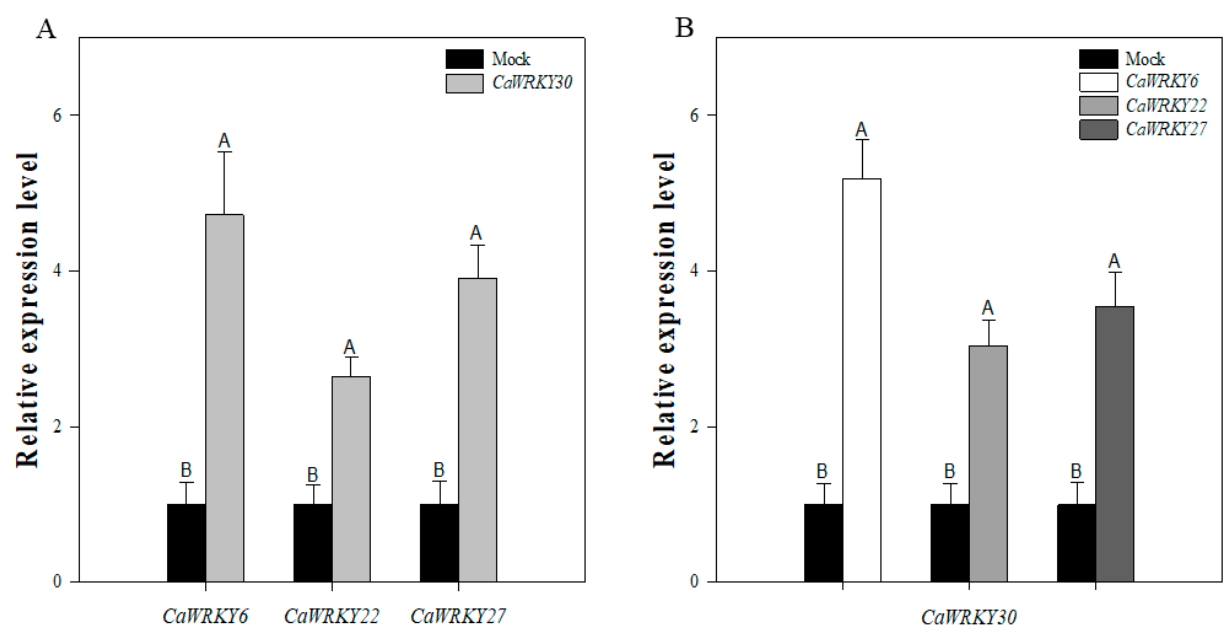

Figure 7. Inter-relationship between CaWRKY30 and CaWRKY6, CaWRKY22, and CaWRKY27. Transcriptional expression level of CaWRKY30 in pepper's leaves transiently over-expressingCaWRKY6, CaWRKY22, and CaWRKY27at 48 hpi (A), Transcriptional expression ofCaWRKY6, CaWRKY22, and CaWRKY27 in pepper's leaves transiently over-expressing CaWRKY30 at 48 hpi (B). Data are means \pm SE from four biological replicates. Error bars are standard errors of the means. Different letters above the bars show significant differences among means.

\section{Discussion}

The WRKY protein is one of the largest families of plant transcription factors (TFs). A group of WRKY TFs family members, reported from Arabidopsis thaliana and Oryza sativa, is known to take part and play a crucial part in plants' immunity regulation. Considerable functional variations among closely structural homologs of WRKYs have been reported [17,48], and functions of WRKY TFs in immunity of non-model plants, such as pepper, is inadequately investigated.

The CaWRKY30, a group III WRKY gene, has been functionally characterized in the current study. Results suggested that CaWRKY30 positively regulates pepper's immunity against RSI, and it is a vital constituent of WRKY web comprising CaWRKY 6, CaWRKY22, CaWRKY27, and CaWRKY40. The entanglement of CaWRKY30 in pepper's immunity is endorsed by pathogen responsive cis elements, including TGA element, TGACG-motif, TATC-box, TATA-box, and W-box present in the promoter region of CaWRKY30. This assumption was consistent with upregulation of transcriptional levels of CaWRKY30 after RSI, and upon exogenously supplied ETH, ABA, and SA [49,50].

Since genes triggered by exposure to a certain stress factor have been often known to be involved in response to that stress [51], we speculated that CaWRKY30 might positively regulate pepper's immunity to RSI. This speculation was supported by the data of silencing of CaWRKY 30 by VIGS, and by CaWRKY30 transient over-expression assay. This curtailed immunity due to loss of function of CaWRKY30 was accompanied by amplified growth of RSI and downregulation of immunity related marker genes like SA dependent CaPR1 [52], CaNPR1 [53], JA related CaDEF1 [54], ABA associated CaABR1 [55], and HR-associated CaHIR1 [56]. On the other hand, HR-like cell death and $\mathrm{H}_{2} \mathrm{O}_{2}$ secretion was triggered by the transient over-expression of CaWRKY30 accompanied with transcriptional up regulation of immunity related marker genes like CaPR1, CaNPR1, CaDEF1, CaABR1, and CaHIR1. This suggests the role of CaWRKY30 as positive regulator of HR-like cell death and pepper's immunity against RSI. Likewise, it has been observed in previous studies that AtWRKY30, a homolog of CaWRKY30 in Arabidopsis, positively regulates plants' immunity against biotic and abiotic stresses $[57,58]$. This can be suggested that CaWRKY30 
is upregulated upon Ralstonia infection, leading to decreased vulnerability of pepper to bacterial pathogen infection.

Phytohormones such as ET, ABA, and SA are essential signaling molecules implicated in response of plants to pathogen attack and high-temperature stress. These are also involved in crosstalk between plants' responses to biotic and abiotic stresses [59]. The SA instigates resistance against biotrophic pathogen, while ET plays a vital role in immunity of plants against necrotrophic pathogens [60]. Generally, synthesis of SA, ET, and JA is coupled with PAMP-triggered immunity (PTI) or effector-triggered-immunity (ETI). These phytohormones can play their role both synergistically and antagonistically based on their concentrations during defense signaling [61]. Synergistic association between these three signaling elements has been detected in PTI, whereas compensatory association has been detected in ETI [62]. The CaWRKY30 was constantly induced by exogenously supplied phytohormones. The known SA, ET, and ABA-dependent immunity-related marker genes such as CaPR1, CaNPR1, CaDEF1, and CaABR1 were downregulated by silencing of CaWRK30; however, they were upregulated by transient over-expression of CaWRKY30 in pepper plants, showing that CaWRKY30 takes part in synergistically mediated defense signaling by SA, ET, and ABA, leading to PTI.

Genome-wide analysis showed the involvement of several WRKY TFs in plant immunity [63-65]. By functional genomics studies, WRKY1 [66], -11 [67], -17 [67], -18 [68], -22 [69], -25 [70], -28 [71], -33 [72], -38 [73], -40 [68], -45 [74], -46 [75], -53 [76], -54 [75], -60 [68], -62 [77], -70 [75], and -75 [78] have been functionally characterized in immunity of Arabidopsis, regulating immunity positively or negatively. These WRKY TFs have been suggested to arrange themselves into a WRKY transcriptional web, comprising of +ve and -ve feedback loops and feed forward modules [12]. However, configuration of these WRKY networks remains poorly understood. In earlier studies we discovered that CaWRKY6, CaWRKY22, CaWRKY27, and CaWRKY40 are positive regulator of pepper's immunity to RSI $[9,44,45]$, while CaWRKY58 is a negative regulator [46]. Current study infers that CaWRKY30 acts as positive regulator in pepper's HR-like cell death, and response of pepper to $R$. solanacearum infection. Transcriptional expression levels of CaWRKY30 were upregulated upon transient over-expression of CaWRKY6, CaWRKY22, CaWRKY27, and CaWRKY40. Conversely, CaWRKY30 transient over-expression upregulated the transcriptional accumulation of CaWRKY6, CaWRKY22, CaWRKY27, and CaWRKY40, suggesting the presence of WRKY TFs networks and +ve feedback loops among CaWRKY6, CaWRKY22, CaWRKY27, and CaWRKY40. Alike +ve feedback loops are supposed to be involved in immunity of plants [47]. The same sort of +ve feedback loop has been present among CaWRKY40 and CaWRKY6, CaWRKY40 and CabZIP63, CaWRKY40 and CaCDPK15. In previous studies, CaWRKY6 [44] and CabZIP63 [16] also have been detected to be involved directly and transcriptionally in the regulation of CaWRKY40 expression against RSI. Keeping in view these results, it can be suggested that CaWRKY40 might be coordinated by various TFs. In conclusion, CaWRKY40 expression is co-regulated by CaWRKY6, CaWRKY22, CaWRKY30, and CabZIP63 upon RSI, and CaWRKY40 expression is regulated by CaWRKY6, and CabZIP63, but not by CaWRKY30 in pepper after exposure to high temperature.

\section{Materials and Methods}

\subsection{Plant Materials and Growth Conditions}

Seeds of pepper cultivar 'Mexi' and Nicotiana benthamiana were obtained from Ayub Agriculture Research Institute, Faisalabad, Pakistan. Pepper and N. benthamiana seeds were planted in plastic pots having soil mix [peat moss and perlite; $2 / 1(v / v)$ ] and kept in a growth room under controlled conditions, i.e., $25{ }^{\circ} \mathrm{C}$ temperature, $60-70 \mu \mathrm{mol}$ photons $\mathrm{m}^{-2} \mathrm{~s}^{-1}$ light intensity, and $70 \%$ relative humidity under $16 \mathrm{~h}$ light $/ 8 \mathrm{~h}$ dark photoperiod.

\subsection{Generation of Vectors}

The gateway-cloning technique was employed to generate vectors. To construct satellite vectors, CaWRKY30 full length ORF (with terminal codon or without terminal 
codon) was cloned into entry vector pDONR207 by using a BP reaction. Afterwards, this construct was transferred into destination vectors pMDC83, CD3687 (HA-tag), CD3688 (Flag-tag), and Pk7WG2 by performing LR reaction to generate vectors for sub-cellular localization and over-expression, respectively.

For the construction of vectors for VIGS, $364 \mathrm{bps}$ fragment in $3^{\prime}$-untranslated region (UTR) of CaWRKY30 was selected and the specificity was confirmed by BLAST against genome sequence in database of CM334 (http:/ / peppergenome.snu.ac.kr/ (accessed on 29 October 2021)) and Zunla-1 (http:/ / peppersequence.genomics.cn/page/species/blast. jsp (accessed on 29 October 2021)). Next, the BP reaction was performed to clone this fragment into pDONR207/201, after this construct was transferred into vector PYL279 by performing LR reaction.

\subsection{Pathogen's Growth and Inoculation Procedures}

We isolated a compatible virulent strain of $R$. solanacearum from pepper plants infected by Ralstonia from Dera Ghazi Khan, Pakistan. The tetrazolium chloride method was used to purify above-ground vascular tissue portion exudates of the pathogen-infected plants $[9,45]$. The isolated $R$. solanacearum was cultivated in SPA medium in thermo control shaker $(200 \mathrm{~g}$ potato, $20 \mathrm{~g}$ sucrose, $3 \mathrm{~g}$ beef extract, $5 \mathrm{~g}$ tryptone, and $1 \mathrm{~L}$ of double-distilled $\mathrm{H}_{2} \mathrm{O}$ ) at $200 \mathrm{rpm}$ and $28^{\circ} \mathrm{C}$ for overnight. Next, cultivated $R$. solanacearum strain was centrifuged at $6500 \mathrm{rpm}$ and $28{ }^{\circ} \mathrm{C}$ for $10 \mathrm{~min}$. Liquid supernatant after centrifugation was poured out, and pellets in the bottom of centrifuge tube were diluted in sterilized $10 \mathrm{mM} \mathrm{MgCl} 2$ solution. Bacterial cell density was adjusted to $10^{8} \mathrm{cfu} \mathrm{ml}^{-1}(\mathrm{OD} 600=0.8)$. To study the effects of RSI on transcriptional levels of CaWRKY30 and on the resistance of pepper plants to $R$. solanacearum attack, the top third leaf of pepper plants was infiltrated with $10 \mu \mathrm{L}$ of $R$. solanacearum solution by a syringe without a needle. Pathogen-treated leaves samples were harvested at specific time points for histochemical staining experiments (e.g., DAB staining or trypan blue staining), electrical conductivity, cfu, and RNA extraction for more experiments. For the phenotype experiment of CaWRKY30-silenced and unsilenced plants, roots were injured by using a glass rod and infiltrated with compatible virulent $R$. solanacearum. Plants were kept in a growth chamber in control conditions at $28 \pm 2{ }^{\circ} \mathrm{C}$ temperature, $60-70 \mu \mathrm{mol}$ photons $\mathrm{m}^{-2} \mathrm{~s}^{-1}$ light intensity, $70 \%$ relative humidity, and under a 16-hlight/8-h dark photoperiod after $R$. solanacearum inoculation. Pictures of phenotype were captured after RSI at specific time intervals.

\subsection{Foliar Application of Phytohormones}

To study the effects of phytohormones, healthy pepper plants were sprayed with $100 \mu \mathrm{M}$ ETH, $100 \mu \mathrm{M}$ ABA, and $1 \mathrm{mM}$ SA at four leaf stages. Mock (control) plants were treated with sterile $\mathrm{ddH}_{2} \mathrm{O}$. Samples treated with phytohormones were collected at desired time points for RNA extraction and for further study.

\subsection{Examination of CaWRKY30 Sub-Cellular Localization}

Agrobacterium cells having 35S:CaWRKY30-GFP or 35S:GFP (control) were cultivated overnight in LB medium possessing corresponding antibiotics. Cultured media was centrifuged at $6500 \mathrm{rpm}$, liquid supernatant was poured out, and the pellet in the bottom was diluted in induction medium (10 mM MES, $10 \mathrm{mM} \mathrm{MgCl2,} \mathrm{pH} \mathrm{5.7,} \mathrm{and} 150 \mu \mathrm{M}$ acetosyringone), and set to OD600 $=0.8$. Agrobacterium containing 35S:CaWRKY30-GFP and 35S:GFP were injected into leaves of $N$. benthamiana by using a syringe without needle. The previously described method was used to do 4,6-diamidino-2-phenylindole staining (DAPI) [9]. At $48 \mathrm{hpt}$, GFP and DAPI fluorescence signals were observed, and pictures were captured by a laser scanning confocal microscope (TCS SP8, Leica, Solms, Germany), with an excitation wavelength of $488 \mathrm{~nm}$ and a $505-530 \mathrm{~nm}$ band-pass emission filter. 


\subsection{Histochemical Staining}

Histochemical staining (Trypan blue and 3, 3'-diaminobenzidine) were carried out as described previously $[9,11,79]$. To perform trypan blue staining assay, leaves of pepper were boiled in trypan blue solution $(10 \mathrm{~mL}$ lactic acid, $10 \mathrm{~mL}$ glycerol, $10 \mathrm{~mL}$ phenol,

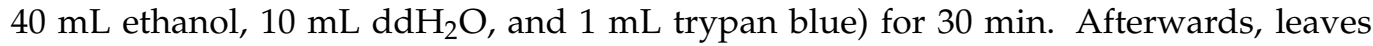
were kept at room temperature for $8 \mathrm{~h}$, immersed into solution of chloral hydrate $(2.5 \mathrm{~g}$ of chloral hydrate dissolved in $1 \mathrm{~mL}$ of distilled water), and de-stained by boiling for $30 \mathrm{~min}$. Chloral hydrate solution was changed multiple times to reduce the background, and then samples were mounted in $70 \%$ glycerol. Leaves were placed in $1 \mathrm{mg} / \mathrm{mL}$ of DAB solution for overnight for DAB staining assay. The DAB stained leaves were boiled in lactic acid:glycerol:absolute ethanol [1:1:3 $(v / v / v)]$ and then de-stained in absolute ethanol for overnight [80]. The pictures of trypan blue and DAB-stained leaves were taken by a camera and by a light microscope (Leica, Wetzlar, Germany).

\subsection{Silencing of CaWRKY30 through Virus-Induced Gene Silencing (VIGS)}

Previously described Tobacco Rattle Virus (TRV)-based virus induced gene silencing (VIGS) system was carried out for CaWRKY30 silencing in pepper plants [47,81,82]. Agrobacterium GV3101 containing PYL192, PYL279-CaWRKY30, PYL279, and PYL279-PDS $\left(\mathrm{OD}_{600}=0.8\right)$ constructs were mixed in a 1:1 ratio. This mix was infiltrated into 2-weeks old pepper plants' cotyledons by a syringe without needle. The infiltrated plants were incubated in a growth chamber for $56 \mathrm{~h}$ under dark at $16^{\circ} \mathrm{C}$ temperature and $45 \%$ relative humidity, and then shifted into a growth room in controlled conditions at $25 \pm 2{ }^{\circ} \mathrm{C}$, 60-70 $\mu \mathrm{mol}$ photons $\mathrm{m}^{-2} \mathrm{~s}^{-1}$, and relative humidity of $70 \%$ under a photoperiod of $16 \mathrm{~h}$ light/8 h dark.

\subsection{CaWRKY30 Transient Over-Expression in Pepper Leaves}

Agrobacterium cells GV3101 containing CaWRKY30-Flag and EV were cultivated in LB medium in a thermo control shaker, having corresponding antibiotics overnight to $\mathrm{OD}_{600}=1.0$. Afterwards, these Agrobacterium GV3101 were centrifuged at $6000 \mathrm{rpm}$ and $28^{\circ} \mathrm{C}$ for $10 \mathrm{~min}$. After centrifugation, liquid supernatant was removed and the solid pellet in the bottom was diluted to $\mathrm{OD}_{600}=0.8$ into induction medium (10 mM MES, pH5.4, $10 \mathrm{mM} \mathrm{MgCl} 2,200 \mu \mathrm{M}$ acetosyringone). This mixture was infiltrated into leaves by a syringe without needle. These transiently over-expressed leaves were observed for HR-like cell death, or samples were collected to perform DAB and trypan blue staining experiments, and for RNA isolation for more experiments.

\subsection{RNA Extraction and Quantitative Real Time RT-PCR}

TRIzol reagent method (Invitrogen) was used to extract total RNA from leaves samples of treated pepper plants and from mock seedlings. Afterwards, Prime Script RT-PCR kit (TaKaRa, Dalian, China) was used to reverse transcribe extracted RNA. Real-time qRT-PCR analysis was carried out to study relative transcriptional levels of targeted marker genes. Data processing was done according to previously described methods [45,82], with specific primers (Supplementary Table S3), using manufacturer's instructions for Bio-Rad Real time PCR system (Bio-Rad, Foster City, CA, USA) and SYBR premix Ex Taq II system (TaKaRa Perfect Real Time).

\subsection{Estimation of ELECTRICAL Conductivity}

Electrical conductivity (ion leakage) was calculated by using the method described previously with small changes $[44,83]$. Six leaf discs ( $4 \mathrm{~mm}$ in width) were obtained by using a hole-puncher. The discs were washed three times with sterilized $\mathrm{ddH}_{2} \mathrm{O}$ and

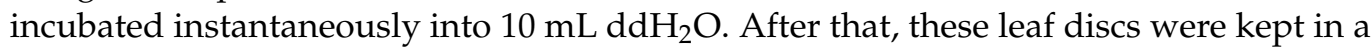
gently shaking $(60 \mathrm{rpm})$ shaker at room temperature for $1 \mathrm{~h}$. A conductivity meter (Mettler Toledo 326 Mettler, Zurich, Switzerland) was used to record data of electrical conductivity. 


\subsection{Immuno Blotting}

A previously described procedure of protein extraction buffer was used to extract protein from pepper leaves [3]. The extracted protein was incubated overnight with antiHA agarose at $4{ }^{\circ} \mathrm{C}$ (Thermo Fisher Scientific, Waltham, MA, USA). Magnetic crack was used to collect beads and washed thrice with Tris buffer saline (TBS) and tween $20(0.05 \%)$. Eluted protein was observed using immuno-blotting and by anti-HA-peroxidase (Abcam, Cambridge, UK).

\section{Conclusions}

In conclusion, we are of the view that CaWRKY30 transcriptionally activates immunity of pepper against RSI. Silencing of CaWRKY30 by VIGS curtailed pepper plants' immunity to bacterial pathogen infection, whereas transient over-expression of CaWRKY30 induced the resistance to $R$. solanacearum. Collectively, the formation of positive feed-back loop and its function in parallel with other TFs is ample evidence of its activation under biotic stress. Additionally, we propose generation of CaWRKY30 stable transgenic pepper plants and CaWRKY30 target protein to comprehensively explicate the role of this gene against bacterial pathogen and also in various metabolic pathways.

Supplementary Materials: The following are available online at https: / /www.mdpi.com/article/ $10.3390 /$ ijms222112091/s1.

Author Contributions: Conceptualization, A.H., M.I.K., M.A., B.S.A., Z.L. and A.T.K.Z.; Data curation, A.H., M.I.K. and A.M.E.-S.; Formal analysis, Z.L. and S.F.; Funding acquisition, A.H., M.I.K., M.A.A.A., M.A., B.S.A., A.M.E.-S. and A.T.K.Z.; Project administration, A.H. and M.I.K.; Resources, A.H.; Validation, S.M.; Visualization, S.M. and S.F.; Writing—original draft, A.H. and M.I.K.; Writingreview \& editing, I.R.N., M.A.A.A., M.A., B.S.A., A.M.E.-S., Z.L., S.F. and A.T.K.Z. All authors have read and agreed to the published version of the manuscript.

Funding: The principal author is highly obliged to the Higher Education Commission of Pakistan for financial assistance to accomplish this study under SRGP grant No. 21-2651/SRGP/HRD/HEC/2020. The current work was funded by Taif University Researchers Supporting Project number (TURSP2020/245), Taif University, Taif, Saudi Arabia. The study was also supported by Development Fund Project of Fujian Agriculture and Forestry University (CXZX2018114, CXZX2020008A, and CXZX2019024G).

Institutional Review Board Statement: Not applicable.

Informed Consent Statement: Not applicable.

Data Availability Statement: All data are within the manuscript and its Supplementary Files.

Conflicts of Interest: The authors declare no conflict of interest.

\section{References}

1. Arif, M.; Atta, S.; Bashir, M.A.; Khan, M.I.; Hussain, A.; Shahjahan, M.; Alwahibi, M.S.; Elshikh, M.S. The impact of FosetylAluminium application timing on Karnal bunt suppression and economic returns of bread wheat (Triticum aestivum L.). PLoS ONE 2021, 16, e0244931.

2. Khalofah, A.; Khan, M.I.; Arif, M.; Hussain, A.; Ullah, R.; Irfan, M.; Mahpara, S.; Shah, R.U.; Ansari, M.J.; Kintl, A. Deep placement of nitrogen fertilizer improves yield, nitrogen use efficiency and economic returns of transplanted fine rice. PLoS ONE 2021, 16, e0247529. [CrossRef] [PubMed]

3. Noman, A.; Hussain, A.; Adnan, M.; Khan, M.I.; Ashraf, M.F.; Zainab, M.; Khan, K.A.; Ghramh, H.A.; He, S. A novel MYB transcription factor CaPHL8 provide clues about evolution of pepper immunity against soil borne pathogen. Microb. Pathog. 2019, 137, 103758. [CrossRef] [PubMed]

4. Hussain, A.; Noman, A.; Khan, M.I.; Zaynab, M.; Aqeel, M.; Anwar, M.; Ashraf, M.F.; Liu, Z.; Raza, A.; Mahpara, S. Molecular regulation of pepper innate immunity and stress tolerance: An overview of WRKY TFs. Microb. Pathog. 2019, 135, 103610. [CrossRef] [PubMed]

5. Reboledo, G.; Agorio, A.d.; Vignale, L.; Batista-García, R.A.; de León, I.P. Transcriptional profiling reveals conserved and species-specific plant defense responses during the interaction of Physcomitrium patens with Botrytis cinerea. Plant Mol. Biol. 2021, 1-21. [CrossRef]

6. Aerts, N.; Mendes, M.P.; van Wees, S.C. Multiple levels of crosstalk in hormone networks regulating plant defense. Plant J. 2021, 105, 489-504. [CrossRef] [PubMed] 
7. Liu, Z.; Shi, L.; Weng, Y.; Zou, H.; Li, X.; Yang, S.; Qiu, S.; Huang, X.; Huang, J.; Hussain, A. ChiIV3 acts as a novel target of WRKY40 to mediate pepper immunity against Ralstonia solanacearum infection. Mol. Plant-Microbe Interact. 2019, 32, $1121-1133$. [CrossRef] [PubMed]

8. ul Haq, M.I.; Maqbool, M.M.; Ali, A.; Farooq, S.; Khan, S.; Saddiq, M.S.; Khan, K.A.; Ali, S.; Khan, M.I.; Hussain, A. Optimizing planting geometry for barley-Egyptian clover intercropping system in semi-arid sub-tropical climate. PLoS ONE 2020, 15, $\mathrm{e} 0233171$.

9. Hussain, A.; Li, X.; Weng, Y.; Liu, Z.; Ashraf, M.F.; Noman, A.; Yang, S.; Ifnan, M.; Qiu, S.; Yang, Y. CaWRKY22 acts as a positive regulator in pepper response to Ralstonia solanacearum by constituting networks with CaWRKY6, CaWRKY27, CaWRKY40, and CaWRKY58. Int. J. Mol. Sci. 2018, 19, 1426. [CrossRef] [PubMed]

10. Mukhtar, M.S.; Liu, X.; Somssich, I.E. Elucidating the role of WRKY27 in male sterility in Arabidopsis. Plant Signal. Behav. 2018, 13, e1363945. [CrossRef] [PubMed]

11. Khan, M.I.; Zhang, Y.; Liu, Z.; Hu, J.; Liu, C.; Yang, S.; Hussain, A.; Ashraf, M.F.; Noman, A.; Shen, L. CaWRKY40b in pepper acts as a negative regulator in response to Ralstonia solanacearum by directly modulating defense genes including CaWRKY40. Int. J. Mol. Sci. 2018, 19, 1403. [CrossRef] [PubMed]

12. Eulgem, T.; Somssich, I.E. Networks of WRKY transcription factors in defense signaling. Curr. Opin. Plant Biol. 2007, 10, 366-371. [CrossRef] [PubMed]

13. Bakshi, M.; Oelmüller, R. WRKY transcription factors: Jack of many trades in plants. Plant Signal. Behav. 2014, 9, e27700. [CrossRef] [PubMed]

14. Xu, Y.-P.; Xu, H.; Wang, B.; Su, X.-D. Crystal structures of N-terminal WRKY transcription factors and DNA complexes. Protein Cell 2020, 11, 208-213. [CrossRef] [PubMed]

15. Chen, X.; Li, C.; Wang, H.; Guo, Z. WRKY transcription factors: Evolution, binding, and action. Phytopathol. Res. 2019, 1, 13. [CrossRef]

16. Shen, L.; Liu, Z.; Yang, S.; Yang, T.; Liang, J.; Wen, J.; Liu, Y.; Li, J.; Shi, L.; Tang, Q. Pepper CabZIP63 acts as a positive regulator during Ralstonia solanacearum or high temperature-high humidity challenge in a positive feedback loop with CaWRKY40. J. Exp. Bot. 2016, 67, 2439-2451. [CrossRef] [PubMed]

17. Schluttenhofer, C.; Yuan, L. Regulation of Specialized Metabolism by WRKY Transcription Factors. Plant Physiol. 2015, 167, 295-306. [CrossRef] [PubMed]

18. Jiang, J.; Ma, S.; Ye, N.; Jiang, M.; Cao, J.; Zhang, J. WRKY transcription factors in plant responses to stresses. J. Integr. Plant Biol. 2017, 59, 86-101. [CrossRef]

19. Yu, K.; Pieterse, C.M.; Bakker, P.A.; Berendsen, R.L. Beneficial microbes going underground of root immunity. Plant Cell Environ. 2019, 42, 2860-2870. [CrossRef]

20. Noman, A.; Liu, Z.; Yang, S.; Shen, L.; Hussain, A.; Ashraf, M.F.; Khan, M.I.; He, S. Expression and functional evaluation of CaZNF830 during pepper response to Ralstonia solanacearum or high temperature and humidity. Microb. Pathog. 2018, 118, 336-346. [CrossRef] [PubMed]

21. Noman, A.; Hussain, A.; Ashraf, M.F.; Khan, M.I.; Liu, Z.; He, S. CabZIP53 is targeted by CaWRKY40 and act as positive regulator in pepper defense against Ralstonia solanacearum and thermotolerance. Environ. Exp. Bot. 2019, 159, 138-148. [CrossRef]

22. Ashraf, M.F.; Yang, S.; Wu, R.; Wang, Y.; Hussain, A.; Noman, A.; Khan, M.I.; Liu, Z.; Qiu, A.; Guan, D.; et al. Capsicum annuum HsfB2a Positively Regulates the Response to Ralstonia solanacearum Infection or High Temperature and High Humidity Forming Transcriptional Cascade with CaWRKY6 and CaWRKY40. Plant Cell Physiol. 2018, 59, 2608-2623. [CrossRef] [PubMed]

23. Wang, L.; Dossa, K.; You, J.; Zhang, Y.; Li, D.; Zhou, R.; Yu, J.; Wei, X.; Zhu, X.; Jiang, S.; et al. High-resolution temporal transcriptome sequencing unravels ERF and WRKY as the master players in the regulatory networks underlying sesame responses to waterlogging and recovery. Genomics 2021, 113, 276-290. [CrossRef]

24. Yang, X.; Zhou, Z.; Fu, M.; Han, M.; Liu, Z.; Zhu, C.; Wang, L.; Zheng, J.; Liao, Y.; Zhang, W.; et al. Transcriptome-wide identification of WRKY family genes and their expression profiling toward salicylic acid in Camellia japonica. Plant Signal. Behav. 2021, 16, 1844508. [CrossRef] [PubMed]

25. Pervez, M.; Babar, M.; Iqbal, J.; Hasnain, M.; Abbas, S.; Shah, S.; Ashraf, M. In Silico Structural and Functional Analysis of Wrky1 and Wrky3 Genes in The Selected Cereal Crops. J. Anim. Plant Sci.-JAPS 2021, 31, 322-341.

26. Liu, Z.-Q.; Liu, Y.-Y.; Shi, L.-P.; Yang, S.; Shen, L.; Yu, H.-X.; Wang, R.-Z.; Wen, J.-Y.; Tang, Q.; Hussain, A.; et al. SGT1 is required in PcINF1/SRC2-1 induced pepper defense response by interacting with SRC2-1. Sci. Rep. 2016, 6, 21651. [CrossRef] [PubMed]

27. Srinivasan, K. Biological Activities of Red Pepper (Capsicum annuum) and Its Pungent Principle Capsaicin: A Review. Crit. Rev. Food Sci. Nutr. 2016, 56, 1488-1500. [CrossRef] [PubMed]

28. Liu, Z.; Shi, L.; Yang, S.; Lin, Y.; Weng, Y.; Li, X.; Hussain, A.; Noman, A.; He, S. Functional and Promoter Analysis of ChiIV3, a Chitinase of Pepper Plant, in Response to Phytophthora capsici Infection. Int. J. Mol. Sci. 2017, 18, 1661. [CrossRef] [PubMed]

29. Ali, M.; Luo, D.-X.; Khan, A.; Haq, S.U.; Gai, W.-X.; Zhang, H.-X.; Cheng, G.-X.; Muhammad, I.; Gong, Z.-H. Classification and Genome-Wide Analysis of Chitin-Binding Proteins Gene Family in Pepper (Capsicum annuum L.) and Transcriptional Regulation to Phytophthora capsici, Abiotic Stresses and Hormonal Applications. Int. J. Mol. Sci. 2018, 19, 2216. [CrossRef] [PubMed]

30. Noman, A.; Liu, Z.; Aqeel, M.; Zainab, M.; Khan, M.I.; Hussain, A.; Ashraf, M.F.; Li, X.; Weng, Y.; He, S. Basic leucine zipper domain transcription factors: The vanguards in plant immunity. Biotechnol. Lett. 2017, 39, 1779-1791. [CrossRef] [PubMed] 
31. Yang, S.; Shi, Y.; Zou, L.; Huang, J.; Shen, L.; Wang, Y.; Guan, D.; He, S. Pepper CaMLO6 Negatively Regulates Ralstonia solanacearum Resistance and Positively Regulates High Temperature and High Humidity Responses. Plant Cell Physiol. 2020, 61, 1223-1238. [CrossRef]

32. Arif, M.; Atta, S.; Bashir, M.A.; Hussain, A.; Khan, M.I.; Farooq, S.; Hannan, A.; Islam, S.U.; Umar, U.U.D.; Khan, M.; et al. Molecular characterization and RSV Co-infection of Nicotiana benthamiana with three distinct begomoviruses. Methods 2020, 183, 43-49. [CrossRef] [PubMed]

33. Brown, D.G.; Allen, C. Ralstonia solanacearum genes induced during growth in tomato: An inside view of bacterial wilt. Mol. Microbiol. 2004, 53, 1641-1660. [CrossRef] [PubMed]

34. Elphinstone, J.G. The Current Bacterial Wilt Situation: A Global Overview, Bacterial Wilt Disease and the Ralstonia Solanacearum Species Complex; APS Press: St. Paul, MN, USA, 2005; pp. 9-28.

35. Argueso, C.T.; Ferreira, F.J.; Kieber, J.J. Environmental perception avenues: The interaction of cytokinin and environmental response pathways. Plant Cell Environ. 2009, 32, 1147-1160. [CrossRef]

36. Choi, J.; Huh, S.U.; Kojima, M.; Sakakibara, H.; Paek, K.-H.; Hwang, I. The Cytokinin-Activated Transcription Factor ARR2 Promotes Plant Immunity via TGA3/NPR1-Dependent Salicylic Acid Signaling in Arabidopsis. Dev. Cell 2010, 19, 284-295. [CrossRef] [PubMed]

37. Shigenaga, A.M.; Berens, M.L.; Tsuda, K.; Argueso, C.T. Towards engineering of hormonal crosstalk in plant immunity. Curr. Opin. Plant Biol. 2017, 38, 164-172. [CrossRef]

38. Silvar, C.; Merino, F.; Díaz, J. Differential activation of defense-related genes in susceptible and resistant pepper cultivars infected with Phytophthora capsici. J. Plant Physiol. 2008, 165, 1120-1124. [CrossRef]

39. Cargnello, M.; Roux, P.P. Activation and Function of the MAPKs and Their Substrates, the MAPK-Activated Protein Kinases. Microbiol. Mol. Biol. Rev. 2011, 75, 50-83. [CrossRef]

40. Boudsocq, M.; Sheen, J. CDPKs in immune and stress signaling. Trends Plant Sci. 2013, 18, 30-40. [CrossRef]

41. Adnan, M.; Islam, W.; Noman, A.; Hussain, A.; Anwar, M.; Khan, M.U.; Akram, W.; Ashraf, M.F.; Raza, M.F. Q-SNARE protein FgSyn8 plays important role in growth, DON production and pathogenicity of Fusarium graminearum. Microb. Pathog. 2019, 140, 103948. [CrossRef]

42. Ross, C.A.; Liu, Y.; Shen, Q.J. The WRKY Gene Family in Rice (Oryza sativa). J. Integr. Plant Biol. 2007, 49, 827-842. [CrossRef]

43. Imran, Q.M.; Hussain, A.; Mun, B.-G.; Lee, S.-U.; Asaf, S.; Ali, M.A.; Lee, I.-J.; Yun, B.-W. Transcriptome wide identification and characterization of NO-responsive WRKY transcription factors in Arabidopsis thaliana L. Environ. Exp. Bot. 2018, 148, 128-143. [CrossRef]

44. Cai, H.; Yang, S.; Yan, Y.; Xiao, Z.; Cheng, J.; Wu, J.; Qiu, A.; Lai, Y.; Mou, S.; Guan, D.; et al. CaWRKY6 transcriptionally activates CaWRKY40, regulates Ralstonia solanacearum resistance, and confers high-temperature and high-humidity tolerance in pepper. J. Exp. Bot. 2015, 66, 3163-3174. [CrossRef]

45. Dang, F.F.; Wang, Y.N.; Yu, L.; Eulgem, T.; Lai, Y.; Liu, Z.Q.; Wang, X.; Qiu, A.L.; Zhang, T.X.; Lin, J. CaWRKY40, a WRKY protein of pepper, plays an important role in the regulation of tolerance to heat stress and resistance to Ralstonia solanacearum infection. Plant Cell Environ. 2013, 36, 757-774. [CrossRef] [PubMed]

46. Wang, Y.; Dang, F.; Liu, Z.; Wang, X.; Eulgem, T.; Lai, Y.; Yu, L.; She, J.; Shi, Y.; Lin, J. C a WRKY 58, encoding a group I WRKY transcription factor of Capsicum annuum, negatively regulates resistance to Ralstonia solanacearum infection. Mol. Plant Pathol. 2013, 14, 131-144. [CrossRef] [PubMed]

47. Shen, L.; Yang, S.; Yang, T.; Liang, J.; Cheng, W.; Wen, J.; Liu, Y.; Li, J.; Shi, L.; Tang, Q.; et al. CaCDPK15 positively regulates pepper responses to Ralstonia solanacearum inoculation and forms a positive-feedback loop with CaWRKY40 to amplify defense signaling. Sci. Rep. 2016, 6, 22439. [CrossRef]

48. Chanwala, J.; Satpati, S.; Dixit, A.; Parida, A.; Giri, M.K.; Dey, N. Genome-wide identification and expression analysis of WRKY transcription factors in pearl millet (Pennisetum glaucum) under dehydration and salinity stress. BMC Genom. 2020, $21,231$. [CrossRef]

49. Mine, A.; Seyfferth, C.; Kracher, B.; Berens, M.L.; Becker, D.; Tsuda, K. The Defense Phytohormone Signaling Network Enables Rapid, High-Amplitude Transcriptional Reprogramming during Effector-Triggered Immunity. Plant Cell 2018, 30, 1199-1219. [CrossRef] [PubMed]

50. Pieterse, C.M.A.; Leon-Reyes, A.; Van der Ent, S.; Van Wees, S.C. Networking by small-molecule hormones in plant immunity. Nat. Chem. Biol. 2009, 5, 308-316. [CrossRef] [PubMed]

51. Kim, B.-M.; Rhee, J.-S.; Jeong, C.-B.; Seo, J.S.; Park, G.S.; Lee, Y.-M.; Lee, J.-S. Heavy metals induce oxidative stress and trigger oxidative stress-mediated heat shock protein (hsp) modulation in the intertidal copepod Tigriopus japonicus. Comp. Biochem. Physiol. Part C Toxicol. Pharmacol. 2014, 166, 65-74. [CrossRef] [PubMed]

52. Sabater-Jara, A.B.; Almagro, L.; Belchí-Navarro, S.; Ferrer, M.Á.; Barceló, A.R.; Pedreño, M.Á. Induction of sesquiterpenes, phytoesterols and extracellular pathogenesis-related proteins in elicited cell cultures of Capsicum annuum. J. Plant Physiol. 2010, 167, 1273-1281. [CrossRef]

53. Barsalobres-Cavallari, C.; Petitot, A.; Severino, F.; Maia, I.; Fernandez, D. Host response profiling to fungal infection: Molecular cloning, characterization and expression analysis of NPR1 gene from coffee (Coffea arabica). Microb. Pathog. Strateg. Combat. Sci. Technol. Educ. 2013, 4, 411-418. 
54. Wang, Q.; Qiu, B.; Li, S.; Zhang, Y.; Cui, X.; Ge, F.; Liu, D. A methyl jasmonate induced defensin like protein from Panax notoginseng confers resistance against Fusarium solani in transgenic tobacco. Biol. Plant. 2019, 63, 797-807. [CrossRef]

55. Choi, D.S.; Hwang, B.K. Proteomics and Functional Analyses of Pepper Abscisic Acid-Responsive 1 (ABR1), Which Is Involved in Cell Death and Defense Signaling. Plant Cell 2011, 23, 823-842. [CrossRef] [PubMed]

56. Choi, H.W.; Kim, Y.J.; Hwang, B.K. The Hypersensitive Induced Reaction and Leucine-Rich Repeat Proteins Regulate Plant Cell Death Associated with Disease and Plant Immunity. Mol. Plant-Microbe Interact. 2011, 24, 68-78. [CrossRef]

57. Scarpeci, T.E.; Zanor, M.I.; Mueller-Roeber, B.; Valle, E.M. Overexpression of AtWRKY30 enhances abiotic stress tolerance during early growth stages in Arabidopsis thaliana. Plant Mol. Biol. 2013, 83, 265-277. [CrossRef]

58. El-Esawi, M.A.; Al-Ghamdi, A.A.; Ali, H.M.; Ahmad, M. Overexpression of AtWRKY30 Transcription Factor Enhances Heat and Drought Stress Tolerance in Wheat (Triticum aestivum L.). Genes 2019, 10, 163. [CrossRef]

59. Berens, M.L.; Berry, H.M.; Mine, A.; Argueso, C.T.; Tsuda, K. Evolution of Hormone Signaling Networks in Plant Defense. Annu. Rev. Phytopathol. 2017, 55, 401-425. [CrossRef] [PubMed]

60. Kunkel, B.N.; Brooks, D.M. Cross talk between signaling pathways in pathogen defense. Curr. Opin. Plant Biol. 2002 , 5, 325-331. [CrossRef]

61. Robert-Seilaniantz, A.; Grant, M.; Jones, J.D. Hormone Crosstalk in Plant Disease and Defense: More than Just Jasmonate-Salicylate Antagonism. Annu. Rev. Phytopathol. 2011, 49, 317-343. [CrossRef] [PubMed]

62. Yuan, M.; Ngou, B.P.M.; Ding, P.; Xin, X.-F. PTI-ETI crosstalk: An integrative view of plant immunity. Curr. Opin. Plant Biol. 2021, 62, 102030. [CrossRef] [PubMed]

63. Pandey, S.P.; Somssich, I.E. The Role of WRKY Transcription Factors in Plant Immunity. Plant Physiol. 2009, 150, 1648-1655. [CrossRef]

64. Rushton, P.J.; Somssich, I.E.; Ringler, P.; Shen, Q.J. WRKY transcription factors. Trends Plant Sci. 2010, 15, 247-258. [CrossRef]

65. Chen, F.; Hu, Y.; Vannozzi, A.; Wu, K.; Cai, H.; Qin, Y.; Mullis, A.; Lin, Z.; Zhang, L. The WRKY Transcription Factor Family in Model Plants and Crops. Crit. Rev. Plant Sci. 2017, 36, 311-335. [CrossRef]

66. Qiao, Z.; Li, C.-L.; Zhang, W. WRKY1 regulates stomatal movement in drought-stressed Arabidopsis thaliana. Plant Mol. Biol. 2016, 91, 53-65. [CrossRef] [PubMed]

67. Ali, M.A.; Azeem, F.; Nawaz, M.A.; Acet, T.; Abbas, A.; Imran, Q.M.; Shah, K.H.; Rehman, H.M.; Chung, G.; Yang, S.H.; et al. Transcription factors WRKY11 and WRKY17 are involved in abiotic stress responses in Arabidopsis. J. Plant Physiol. 2018, 226, 12-21. [CrossRef] [PubMed]

68. Liu, Z.-Q.; Yan, L.; Wu, Z.; Mei, C.; Lu, K.; Yu, Y.-T.; Liang, S.; Zhang, X.-F.; Wang, X.-F.; Zhang, D.-P. Cooperation of three WRKY-domain transcription factors WRKY18, WRKY40, and WRKY60 in repressing two ABA-responsive genes ABI4 and ABI5 in Arabidopsis. J. Exp. Bot. 2012, 63, 6371-6392. [CrossRef] [PubMed]

69. Hsu, F.-C.; Chou, M.-Y.; Chou, S.-J.; Li, Y.-R.; Peng, H.-P.; Shih, M.-C. Submergence Confers Immunity Mediated by the WRKY22 Transcription Factor in Arabidopsis. Plant Cell 2013, 25, 2699-2713. [CrossRef] [PubMed]

70. Doll, J.; Muth, M.; Riester, L.; Nebel, S.; Bresson, J.; Lee, H.-C.; Zentgraf, U. Arabidopsis thaliana WRKY25 Transcription Factor Mediates Oxidative Stress Tolerance and Regulates Senescence in a Redox-Dependent Manner. Front. Plant Sci. 2020, $10,1734$. [CrossRef] [PubMed]

71. Zhao, L.; Cai, H.; Su, Z.; Wang, L.; Huang, X.; Zhang, M.; Chen, P.; Dai, X.; Zhao, H.; Palanivelu, R.; et al. KLU suppresses megasporocyte cell fate through SWR1-mediated activation of WRKY28 expression in Arabidopsis. Proc. Natl. Acad. Sci. USA 2018, 115, E526-E535. [CrossRef] [PubMed]

72. Birkenbihl, R.P.; Diezel, C.; Somssich, I.E. Arabidopsis WRKY33 Is a Key Transcriptional Regulator of Hormonal and Metabolic Responses toward Botrytis cinerea Infection. Plant Physiol. 2012, 159, 266-285. [CrossRef] [PubMed]

73. Kalachova, T.; Leontovyčová, H.; Iakovenko, O.; Pospíchalová, R.; Maršík, P.; Klouček, P.; Janda, M.; Valentová, O.; Kocourková, D.; Martinec, J. Interplay between phosphoinositides and actin cytoskeleton in the regulation of immunity related responses in Arabidopsis thaliana seedlings. Environ. Exp. Bot. 2019, 167, 103867. [CrossRef]

74. Chen, L.; Xiang, S.; Chen, Y.; Li, D.; Yu, D. Arabidopsis WRKY45 Interacts with the DELLA Protein RGL1 to Positively Regulate Age-Triggered Leaf Senescence. Mol. Plant 2017, 10, 1174-1189. [CrossRef] [PubMed]

75. Chen, J.; Nolan, T.; Ye, H.; Zhang, M.; Tong, H.; Xin, P.; Chu, J.; Chu, C.; Li, Z.; Yin, Y. Arabidopsis WRKY46, WRKY54 and WRKY70 Transcription Factors Are Involved in Brassinosteroid-Regulated Plant Growth and Drought Response. Plant Cell 2017, 29, 1425-1439. [CrossRef] [PubMed]

76. Xie, Y.; Huhn, K.; Brandt, R.; Potschin, M.; Bieker, S.; Straub, D.; Doll, J.; Drechsler, T.; Zentgraf, U.; Wenkel, S. REVOLUTA and WRKY53 connect early and late leaf development in Arabidopsis. Development 2014, 141, 4772-4783. [CrossRef] [PubMed]

77. Fukushima, S.; Mori, M.; Sugano, S.; Takatsuji, H. Transcription Factor WRKY62 Plays a Role in Pathogen Defense and HypoxiaResponsive Gene Expression in Rice. Plant Cell Physiol. 2016, 57, 2541-2551. [CrossRef] [PubMed]

78. Rishmawi, L.; Pesch, M.; Jüngst, C.; Schauss, A.C.; Schrader, A.; Hülskamp, M. Non-Cell-Autonomous Regulation of Root Hair Patterning Genes by WRKY75 in Arabidopsis. Plant Physiol. 2014, 165, 186-195. [CrossRef] [PubMed]

79. Choi, D.S.; Hwang, I.S.; Hwang, B.K. Requirement of the Cytosolic Interaction between Pathogenesis-Related Protein10 and Leucine-Rich Repeat Protein1 for Cell Death and Defense Signaling in Pepper. Plant Cell 2012, 24, 1675-1690. [CrossRef] [PubMed]

80. Korasick, D.; McMichael, C.; Walker, K.A.; Anderson, J.C.; Bednarek, S.Y.; Heese, A. Novel Functions of Stomatal CytokinesisDefective 1 (SCD1) in Innate Immune Responses against Bacteria. J. Biol. Chem. 2010, 285, 23342-23350. [CrossRef] [PubMed] 
81. Azevedo, C.; Sadanandom, A.; Kitagawa, K.; Freialdenhoven, A.; Shirasu, K.; Schulze-Lefert, P. The RAR1 Interactor SGT1, an Essential Component of R Gene-Triggered Disease Resistance. Science 2002, 295, 2073-2076. [CrossRef]

82. Liu, Z.; Shi, L.; Liu, Y.; Tang, Q.; Shen, L.; Yang, S.; Cai, J.; Yu, H.; Wang, R.; Wen, J.; et al. Genome-wide identification and transcriptional expression analysis of mitogen-activated protein kinase and mitogen-activated protein kinase kinase genes in Capsicum annuum. Front. Plant Sci. 2015, 6, 780. [CrossRef] [PubMed]

83. Hwang, I.S.; Hwang, B.K. The Pepper Mannose-Binding Lectin Gene CaMBL1 Is Required to Regulate Cell Death and Defense Responses to Microbial Pathogens. Plant Physiol. 2011, 155, 447-463. [CrossRef] [PubMed] 